\title{
INFLUENCE DES VARIATIONS SAISONNIÈRES DE LA COMPOSITION DU LAIT DE CHĖVRE SUR LE RENDEMENT EN FROMAGE. CONSÉQUENCES PRATIQUES POUR LA SÉLECTION
}

\author{
G. RICORDEAU et G. MOCQUOT \\ avec la collaboration de J. Boulloon (Coopérative "Le l'élardon-des-Cévenues ", Lozère); \\ F. Blanc-Patin et R. Jeuner (Station expérimentale laitière, I. N. R. A, Poligny); \\ A. Portmann (Laboratoire de Technologie laitiere, I. N. R. A, Surgères) \\ Station centrale de Génétique animale, \\ Station centrale de Recherches laitières et de Technologie des Produits animaux, \\ Centre national de Recherches zootechniques, 78 - Jouy-en-Josas \\ Institut national de la Recherche agronomique
}

SOMMAIRE

Cette étude a été entreprise dans 2 laiteries (en 1965 dans la laiterie A, de 1962 à 65 dans la laiterie B) traitant uniquement du lait de chèvre. Elle a permis d'analyser les variations saisonnières de la teneur du lait en matière grasse, matières azotées (totales et coagulables), calcium et de déterminer (par la méthode des régressions multiples) l'influence respective de ces constituants sur le rendement en fromage.

Le lait de chèvre est aussi riche en calcium ( $1,17 \mathrm{~g} / \mathrm{kg}$ de lait) que le lait de vache. Par contre, il est relativement pauvre en matières azotées totales et coagulables $(29,38 \mathrm{~g}$ de MAT par kg de lait, dont 69,I p. Ioo seulement sont coagulables).

Dans les 2 laiteries, le taux de matières azotées totales constitue la meilleure base d'estimation du rendement en fromage puisqu'il explique seul 75 p. 100 environ des variations de ce rendement ; l'influence du taux butyreux est peu ou pas significative.

Il existe entre les 2 laiteries, des différences dues à la méthode de fabrication. Ainsi, l'égouttage préalable en sac, pratiqué dans la laiterie $A$, réduit la variation du poids moyen des fromages et permet d'obtenir une meilleure estimation des rendements.

Pour améliorer génétiquement la valeur fromagère des laits de chèvre, il apparaît nécessaire de remplacer le " contrôle beurrier ", pratiqué actuellement, par un contrôle de la richesse en protéines.

\section{INTRODUCTION}

En France, l'élevage de la chèvre est axé sur la production fromagère, puisqu'en dehors du lait consommé par les jeunes, toute la production laitière est transformée en fromage (FATOUX, Ig65).

Si la quantité de lait doit être le principal caractère à sélectionner, on ne peut négliger sa composition. En effet, les chèvres appartenant aux races exploitées en 
France (Alpine chamoisèe, Saanen, Poitevine) donnent déjà en moyenne un lait plus pauvre que le lait de vache. I a sélection sur la seule quantité risquerait donc, à terme, de donner naissance à des souches produisant beaucoup de lait, mais un lait très pauvre.

Pour que le contrôle de la composition du lait soit efficace, il faut avoir précisé ce que l'on entend par "valeur fromagère " de ce lait. Si de nombreuses études ont été consacrées à ce problème en ce qui concerne le lait de vache (cf. notamment Mocouot, RicordeAu, Aurior, ig63; Mocquot et al., I963), on possède peu d'informations sur le lait et le fromage de chèvre, à part quelques études analytiques (KNOWles et WATKIn, I938; LABUSSI⿳亠口冋e, AlAIS et BeJAMBEs, I959; GaIrLARD et Huxi, I965; Portmaxx et al. Ig66; Fatoux, ig66).

La présente étude a pour but :

I. de connaître la teneur moyenne du lait de chèvre en matière grasse, protéines totales, protéines coagulables et calcium ;

2. d'établir l'importance relative des principaux constituants du lait dans la détermination du rendement en fromage ;

3. de tirer les conclusions afin d'orienter la sélection vers la production de laits présentant la composition la plus favorable.

\section{MATÉRIEL ETT MÉTHODES}

Cette étude a été entreprise dans 2 laiteries traitant uniquement du lait de chèvre et fabriquant un même type de fromage.

- en 1965, dans la laiterie $A$ (Anjouin, Indre) : 40 observations.

- de 1962 à 1965, dans la laiterie B (Moissac, Lozère) : 106 observations.

\section{I - Fabricalion des fromages}

a) Laiterie $A$.

Le lait des 2 traites (soir et matin) est rassemblé dans un bac puis il est emprésuré ì $23^{\circ} \mathrm{C}$ dans des bassines de 50 litres $(20 \mathrm{ml}$ de présure de force $\mathrm{I} / 2500 \mathrm{par}$ hl de lait). Vingt-quatre heures après l'emprésurage, le caillé est mis en sacs pour réaliser un début d'égout tage qui est plus ou moins long

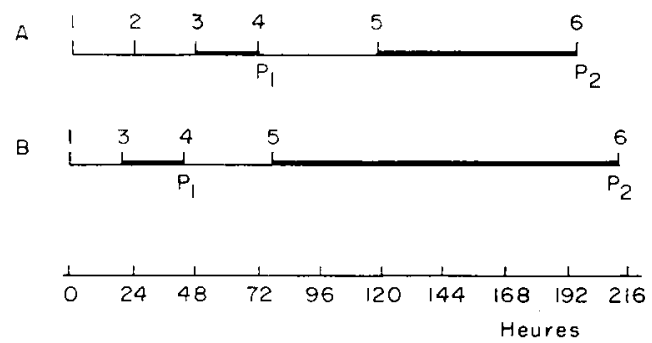

FIGURE I. - Schéma de fabrication dans les laiteries $A$ el $B$

I : emprésurage, 2 : pré-égouttage en sacs, $3:$ mise en moules, $4:$ démoulage, $5:$ entrée au haloir, $\mathrm{P}_{1}$ : pesée au démoulage, $\mathrm{P}_{2}$ : pesée à \&̀ jours environ.

suivant la saison ( 6 à ${ }_{5} 5$ heures). Ia mise en moules a lieu le $3^{\mathrm{e}}$ jour - le $4^{\mathrm{e}}$ jour, démoulage (stade $P_{1}$ ) salage et pose sur claies d'égouttage - le $5^{\mathrm{e}}$ jour mise sur paillons - le $6^{\mathrm{e}}$ jour, passage sur claies nues et entrée au haloir -- les $7^{\mathrm{e}}, 8^{\mathrm{e}}$ et $9^{\mathrm{e}}$ jours, retournements des fromages. 


\section{b) Laiterie $B$.}

Le lait des 2 traites est rassemblé dans un bac, additionné de levain lactique ( 1 à 3 p. roo) puis il est emprésuré à $26^{\circ} \mathrm{C}$ dans des bassines de 50 et I00 lit res ( $12 \mathrm{ml}$ de présure au $\mathrm{I} / \mathrm{IO} 000 \mathrm{par}$ hl de lait). La mise en moules a lieu 20 heures après l'emprésurage. I.es fromages sont ensuite retournés et salés (sur une face) puis démoulés (et salés sur la deuxième face) 23 heures après le début de la mise en moules. L'égout tage du fromage se poursuit à la température ambiante pendant 30 à 48 heures après quoi on procède à l'affinage au haloir pendant au moins 5 jours. En fait, au cours des 2 dernières années ( 1964 et 65 ) la période d'égouttage a été réduite d'environ 24 heures, la période d'affinage au haloir étant prolongée d'autant. La figure I permet de comparer schématiquement. les 2 types de fabrication $A$ et $B$.

\section{II - Prélèvements et pesées}

Les quantités journalières de lait traitées par les 2 laiteries varient de 400 à 4 ooo litres suivant la saison. Les prélevements et les pesées sont faits une fois par semaine.

Laiterie $A$ : pesée de $50 \mathrm{~kg}$ de lait représentatif de l'ensemble du lait de mélange livré à la laiterie et prélèvement d'un échantillon ; sérum prélevé sous les sacs et à la goulotte des tables d'égouttage; pesée de la totalité des fromages correspondants aux $50 \mathrm{~kg}$ de lait mis en cuuvre.

Laiterie $B$ : pesée, à la réception, de l'ensemble du lait traité le jour du contrôle ; prélèvement des échantillons de lait dans le bac de stockage après homogénéisation; sérum recueilli en 2 ou 3 fois à la goulotte des tables d'égouttage ; pesée des fromages correspondant à la totalité du lait mis en ouvre.

Dans les 2 laiteries : pesée des fromages au démoulage $\left(\mathrm{P}_{1}\right)$ et à 8 jours environ après l'emprésurage $\left(\mathrm{P}_{2}\right)$; chaque semaine, mais uniquement en 1965 , prélèvement de 3 fromages en vue des analyses concernant la composition.

$$
\text { III -.. Analyses }
$$

Les analyses ont été effectuées par la Station expérimentale laitière de Poligny (Jura) sur les échantillons de lait, de sérum et de fromage ( ${ }^{1}$.

Les analyses de lait et de sérum concernaient les éléments suivants : le taux de matière grasse déterminé par le procédé Gerber; le taux de matières azotées déterminé par la méthode Kjeldahl (teneur en $\mathrm{N} \times 6,38$ ); le taux de calcium déterminé par la méthode de Jenness (I953).

En ce qui concerne les fromages, on dosait l'extrait sec, la teneur en matières azotées $(\mathrm{N} \times 6,38)$ et les cendres. Nous n'avons pas eu la possibilité de déterminer directement la teneur en graisse du fromage. Une estimation de cette teneur a été obtenue en calculant le rapport que nous avons appelé $\mathrm{D} / \mathrm{S}$ :

$$
\frac{\text { (extrait } \sec \text { total })- \text { (matières azotées }+ \text { cendres) }}{\text { extrait sec total }}
$$

La proportion de matières azotées coagulables dans les matières azotées totales (MAC p. 10o) a été calculée d'après la formule de MocQuOT et ALAIs (1950) dans laquelle TB est la teneur en matière grasse du lait et ' $\mathrm{TB}^{\prime}$ celle du lactosérum ; TP est la teneur en protéines du lait et ' $\mathrm{TP}^{\prime}$ celle du lactosérum $\left({ }^{2}\right)$.

avec

$$
\begin{aligned}
& \mathrm{MAC} \text { p. } 100=100-100 \mathrm{~K} \frac{\mathrm{TP}^{\prime}}{\mathrm{TP}} \\
& \mathrm{K}=\frac{900-0,9 \mathrm{~TB}-\mathrm{I}, 45 \mathrm{TP}}{900-0,9 \mathrm{~TB}^{\prime}-\mathrm{I}, 45 \mathrm{TP}}
\end{aligned}
$$

Le rendement en fromage est représenté par le poids de fromage obtenu à partir de too $\mathrm{kg}$ de lait mis en œuvre. Pour tenir compte des variations observées en pratique dans la teneur en extrait sec des fromages, nous avons appliqué la correction proposée par MAUBOIs et MocQuoT (I967) dans laquelle $\mathrm{ES}_{1}$ exprime la quantité d'extrait sec contenue dans $\mathrm{ooo} \mathrm{g}$ de fromage obtenu, $\mathrm{ES}_{2}$ la quantité d'extrait sec contenue dans $100 \mathrm{~g}$ de fromage "moyen " (le fromage moyen est celui dont la teneur en extrait sec est égale à la moyenne de toutes les fabrications) et $s$ la quantité d'extrait sec contenue dans Ioo $\mathrm{g}$ de lactosérum. De cette façon on peut ramener tous les fromages à un seul et même fromage dont la teneur en extrait sec est bien déterminée.

(1) Pour réduire certains risques d'erreurs, dues par exemple au barattage des échantillons de lait au cours cu transport, le dosage de la matière grasse du lait a été effectué sur place, dans lés 2 laiteries, dès le début de 1965.

(2) $\mathrm{TB}, \mathrm{TB}^{\prime}, \mathrm{TP}, \mathrm{TP}^{\prime}$ sont exprimés en $\mathrm{g}$ par $\mathrm{kg}$ de lait. 
Pour roo g de fromage, la correction $\mathrm{C}$ est égale à

$$
\begin{gathered}
\mathrm{C}=\operatorname{Ioo} \frac{\left(\mathrm{ES}_{1}-\mathrm{ES}_{2}\right)}{\left(\mathrm{ES}_{2}-\mathrm{s}\right)} \\
\mathrm{IV}-\text { Symboles }
\end{gathered}
$$

Dans les tableaux nous utiliserons les symboles suivants :

TB : taux butyreux du lait en $\mathrm{g}$ de graisse par $\mathrm{kg}$ de lait $(\mathrm{g} / \mathrm{kg})$.

$\mathrm{TP}$ : taux de protéines du lait en $\mathrm{g} / \mathrm{kg}$.

$\mathrm{TCa}$ : taux de calcium du lait en $\mathrm{g} / \mathrm{kg}$.

MAC : matières azotées coagulables en p. ioo des matières azotées totales.

$R_{1}$ : rendement en fromage au démoulage.

$\mathrm{R}_{2}$ : rendement en fromage à 8 jours environ.

$\mathrm{R}_{2}^{\prime}$ : rendement en fromage corrigé pour l'extrait sec (ES à 8 jours).

$\mathrm{ES}$ : extrait sec pour $100 \mathrm{~g}$ de fromage prélevé à 8 jours.

$\mathrm{D} / \mathrm{S}:$ matière grasse du fromage en p. Ioo de l'ES.

$\mathrm{P}_{1}$ : poids moyen des fromages frais.

$\mathbf{P}_{2}$ : poids moyen des fromages secs.

$\mathrm{P}_{1-2}$ : perte de poids des fromages entre le démoulage et 8 jours.

$Z_{1}$ : poids de fromage frais par $\mathrm{kg}$ de matière grasse du lait mis en ouvre.

$\mathrm{Z}_{2}$ : poids de fromage sec par $\mathrm{kg}$ de matière grasse du lait mis en œuvre.

$\mathrm{Z}_{2}^{\prime}$ : poids de fromage à 8 jours (par $\mathrm{kg}$ de matière grasse du lait) corrigé pour l'extrait sec.

Dans les analyses de régression multiple :

$\mathbf{F}$ : test relatif à toutes les variables (variance due à la régression/variance résiduelle) avec $v_{1}=k$ et $v_{2}=n-k-1$ ( $k$ étant le nombre de variables indépendantes).

$\mathrm{R}^{2}$ : carré du coefficient de corrélation.

$s^{\prime}{ }_{R}$ : estimation de l'écart-type résiduel du rendement.

$t^{2}:$ test relatif ì chaque coefficient de régression $b_{i}\left(t^{2}=b_{i} / s^{2} b_{i}\right)$.

\section{RÉSUL,TATS}

\section{I - Paramètres statistiques}

a) Moyennes.

Les laits de la laiterie $B$ sont plus riches que ceux de la laiterie $A$, surtout en ce qui concerne la matière grasse : la différence est en effet peu sensible pour la teneur en protéines, la teneur en calcium et la proportion de matières azotées coagulables (tabl. I, fig. 2). En moyenne, la laiterie A a utilisé un lait dans lequel le rapport protéines/graisse est plus élevé $(0,95)$ que celui de la laiterie $B(0,85)$ et a obtenu des rendements supérieurs. Cependant, les conditions de fabrication et de pesées ne sont pas tout à fait comparables à celles de la laiterie B.

\section{b) Variabilité.}

Le taux butyreux varie de la même façon dans les 2 laiteries, mais l'amplitude de variation d $\mathbf{u}$ taux de protéines est moins importante dans la laiterie $B$. Dans cette laiterie, les essais fromagers ont été effectués sur tune période plus réduite (avril à octobre) ce qui n'a pas permis de prendre en considération les laits de début et surtout de fin de lactation dont la richesse est élevée (d'octobre à décembre, le taux de protéines passe de $3 \mathrm{I}$ à $36 \mathrm{~g}$ dans la laiterie $\mathrm{A})$.

Le taux de calcium est pratiquement aussi variable que le taux butyreux ou le taux de protéines.

La proportion de matières azotées coagulables est le paramètre le moins variable (coefficient de variation de $\mathrm{I}, 9$ et $\mathrm{I}, 7$ p. IOo). 
Le poids moyen des fromages frais $\left(P_{1}\right)$ est nettement plus variable dans la laiterie $B$ que dans la laiterie $A$. Ceci peut s'expliquer par le mode de fabrication différent. Ainsi, dans la laiterie $B$, le moulage est effectué dans un nombre de moules constant (ou déterminé à l'avance) ; de ce fait, lorsque le rendement du lait en fromage augmente, le poids de chaque fromage augmente lui aussi. Dans la laiterie A, au contraire, le caillé qui a subi un début d'égouttage est réparti dans des moules recevant toujours la même quantité de caillé. Par conséquent, lorsque le rendement augmente, on remplit plus de moules, mais les fromages ont une taille et un poids assez constants. Nous verrons ultérieurement l'importance de cette observation.

La perte de poids des fromages entre le démoulage et 8 jours $\left(\mathrm{P}_{\mathbf{L}_{-2}}\right)$ est le paramètre le plus variable, ce qui correspond bien à l'avis des fromagers suivant lequell'égouttage et le séchage des fromages de petit format sont des opérations difficiles à contrôler.

\section{TABLEAU I}

Résultats d'ensemble

\begin{tabular}{|c|c|c|c|c|c|c|}
\hline & \multicolumn{3}{|c|}{ Laiterie $\mathrm{A}(n=40)$} & \multicolumn{3}{|c|}{ Laiterie B $(n=106)$} \\
\hline & $\bar{x}$ & $\sigma$ & $\mathrm{CV}$ & $\bar{x}$ & $\sigma$ & $\mathrm{CV}$ \\
\hline $\mathrm{TB}$ & 30,55 & 1,64 & 5,36 & 34,93 & 1,81 & 5,18 \\
\hline $\mathrm{TP}$ & 29,00 & 2,85 & 9,82 & 29,76 & 1,43 & 4,80 \\
\hline $\mathrm{TCa}$ & 1,160 & 0,093 & 8,01 & 1,177 & 0,068 & 5,77 \\
\hline $\mathrm{TP} / \mathrm{TB}$ & 0,949 & 0,077 & 8,13 & 0,854 & 0,056 & 6,51 \\
\hline$\overline{\mathrm{R}_{1}}$ & 18,81 & 1,88 & 10,00 & 17,77 & 1,72 & 9,67 \\
\hline $\mathrm{R}_{2}$ & 14,30 & 2,03 & 14,19 & 11,86 & 1,27 & 10,70 \\
\hline$Z_{1}$ & 6,12 & 0,49 & 8,00 & 5,10 & 0,49 & 9,61 \\
\hline $\mathrm{Z}_{2}$ & 4,68 & 0,56 & 11,95 & 3,40 & 0,39 & 11,45 \\
\hline MAC & 68,68 & 1,31 & 1,90 & 69,63 & 1,17 & 1,68 \\
\hline $\mathrm{P}_{1}$ & 118 & 6,6 & 5,62 & 141 & 10,6 & 7,51 \\
\hline $\mathrm{P}_{2}$ & 90 & 8,4 & 9,35 & 94 & 9,3 & 9,85 \\
\hline$P_{1-2}$ & 28 & 6,8 & 24,56 & 47 & 11,0 & 23,46 \\
\hline ES & 42,8 & 2,2 & 5,16 & $53,5^{*}$ & & \\
\hline $\mathrm{R}_{2}^{\prime}$ & 14,17 & 1,92 & $13,5^{\prime} 4$ & & & \\
\hline $\mathrm{Z}_{2}^{\prime}$ & 4,64 & 0,43 & 9,27 & & & \\
\hline $\mathrm{D} / \mathrm{S}$ & 57,8 & 0,84 & 14,54 & $58,3^{*}$ & & \\
\hline
\end{tabular}

* Estimation portant uniquement sur 25 observations. 


\section{II - Evolution saisonnière de la composition du lait}

I. Bien que les analyses concernent des laits d'assez grand mélange (400 à 4000 1), le taux butyreux apparaît très variable d'une semaine à l'autre (fig. 2). C'est là un point important à souligner. En effet, indépendamment de toute autre considération, il apparaît difficile de baser la valeur fromagère du lait de chèvre sur un composant qui varie aussi largement sur une courte période : l'écart moyen

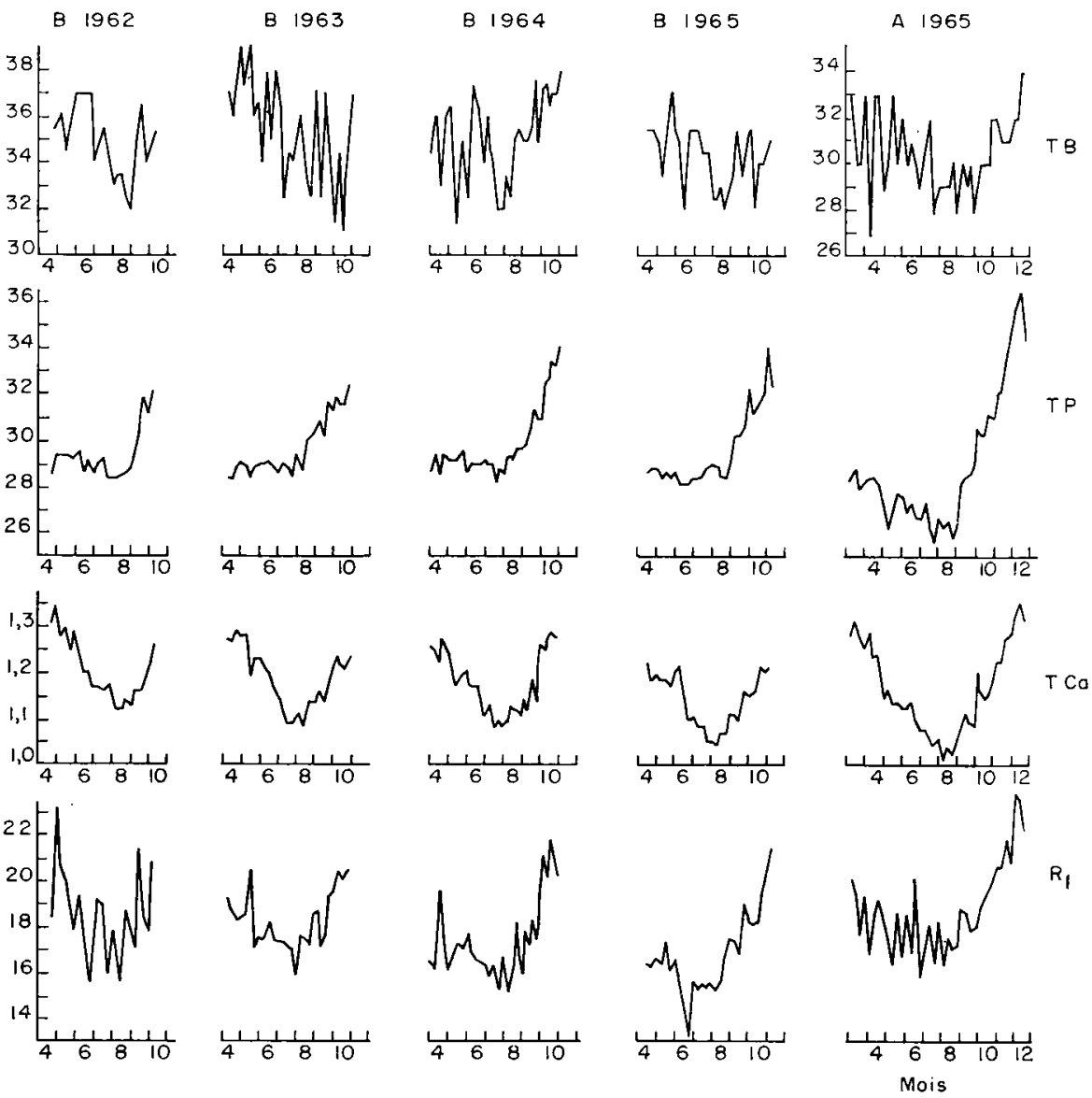

FIGURE 2. - Évolution de la composition du lait de mélange dans les 2 laiteries en fonction du mois et de l'année de contróle

4 années pour la laiterie $\mathrm{B}$, I année pour la laiterie $\mathrm{A}$.

de haut en bas : taux butyreux, taux de protéines, taux de calcium et rendement au démoulage.

en valeur absolue entre 2 contrôles successifs est de $I, 5 \mathrm{~g}$ pour la laiterie $\mathrm{A}$ ( $n=39$, amplitude de $5 \mathrm{~g}$ ) et de $\mathrm{I}, 7 \mathrm{~g}$ pour la laiterie $\mathrm{B}(n=\mathrm{ro2}$, amplitude de $5 \mathrm{~g}$ ).

Dans l'ensemble, le taux butyreux décroît jusqu'au mois d'octobre puis augmente ensuite ; la variabilité entre contrôles rend cependant difficile l'étude des variations saisonnières de la richesse en matière grasse des laits de mélange. 
2. Le taux de protéines est très stable d'un contrôle à l'autre : il reste constant ou diminue légèrement jusqu'en juillet-août puis augmente rapidement jusqu'en décembre (laiterie $\mathrm{A}$ ).

3. Le taux de calcium a également un mouvement saisonnier rigide : diminution de février à juillet puis accroissement comme pour le lait de vache (AURIOL et Mocquot, I962). L'évolution du taux de calcium en sens inverse des variations de la durée d'éclairement incite à penser que le taux de calcium est en partie sous l'influence de la lumière.

4. La proportion de matières azotées coagulables est peu variable : l'analyse de la variance de ce paramètre effectuée à partir de l'ensemble des données des 2 laiteries montre que la variation saisonnière observée (minimum en juillet-août) n'est pas significative (tabl. 2).

TABIEAU 2

Variations saisonnières de la proportion de matières asotées coagulables (MAC p. 100)

\begin{tabular}{|c|c|c|c|c|c|c|c|c|c|c|}
\hline Laiterie & Mars & Avril & Mai & Juin & Juillet & Août & Sept. & Oct. & Nov. & Déc. \\
\hline A & 69,0 & 69,3 & 68,5 & 69,0 & 67,7 & 67,2 & 69,1 & 68,9 & 69,6 & 68,7 \\
\hline$B$ & - & $70,,_{k}^{\prime}$ & 69,6 & 68,9 & 69,3 & 69,0 & 69,7 & 70,6 & 71,4 & - \\
\hline
\end{tabular}

\section{III - Etude statistique des relations entre le rendement en fromage et les principaux composants du lait}

Le tableau 3 représente la matrice des corrélations entre les différentes variables. Le rendement en fromage est influencé par la richesse du lait en protéines, en matière grasse, en calcium et par la proportion de matières azotées coagulables. Le rendement est également en corrélation positive avec le poids moyen des fromages et en corrélation négative avec l'extrait sec des fromages.

Pour déterminer l'influence respective des principaux éléments de la composition du lait, nous avons effectué l'analyse de régression multiple (tabl. 4) entre les variables exprimant le rendement d'une part (rendement frais, à 8 jours et rendement corrigé à 8 jouts pour la teneur en extrait sec des fromages) et les 4 "variables indépendantes "d'autre part (taux de protéines, taux butyreux, taux de calcium et proportion de matières azotées coagulables).

Le coefficient de corrélation multiple est supérieur à $0,89\left(R^{2} \geqslant 0,79\right)$ pour la laiterie $A$, il est de $0,80\left(R^{2}=0,64\right)$ pour la laiterie $B$ en ce qui concerne le rendement frais.

Dans le tableau 4 nous avons calculé la signification des coefficients de régression pour tester l'importance des différentes variables. Seule, l'influence du taux de protéines est très significative dans les 2 laiteries. Dans la laiterie $B$, le taux de cal- 


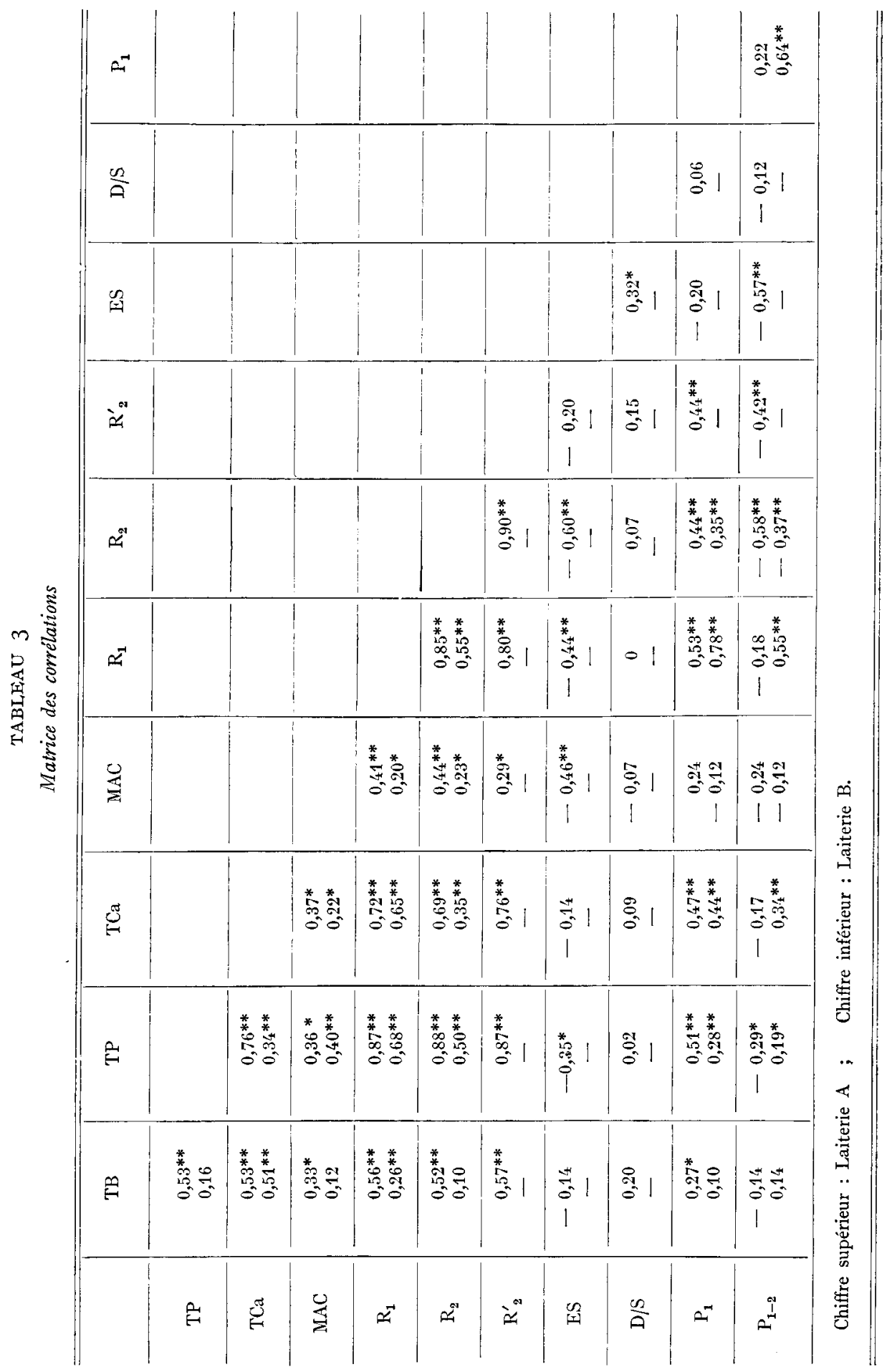


cium présente également une influence hautement significative. Dans tous les cas, le coefficient de régression correspondant au taux butyreux n'est pas significatif, ainsi que celui correspondant à la proportion de matières azotées coagulables.

\section{TABLEAU 4}

Analyse de la régression multiple à 4 ou 5 variables indépendantes

Signification des coefficients (test $t^{2}$ )

\begin{tabular}{|c|c|c|c|c|c|c|}
\hline \multirow{2}{*}{ Variable } & \multicolumn{4}{|c|}{ Laiterie A } & \multicolumn{2}{|c|}{ Laiterie B } \\
\hline & $\mathrm{R}_{1}$ & $\mathrm{R}_{2}$ & $\mathrm{R}_{2}^{\prime}$ & $\mathbf{R}_{2}$ & $\mathrm{R}_{1}$ & $\mathrm{R}_{2}$ \\
\hline TB.. & 1,4 & 0,3 & 2,0 & 1,3 & 1,5 & 1,0 \\
\hline TP... & $33,8 * *$ & $40,2 * *$ & $30,0^{* * *}$ & $35,0^{* *}$ & $53,5 * *$ & $12,2^{* *}$ \\
\hline $\mathrm{TCa}$ & 0,5 & 0 & 3,4 & 2,8 & $53,0^{* *}$ & $6,8^{* *}$ \\
\hline MAC & 0,9 & 2,4 & 0,8 & 0,2 & 2,5 & 0,3 \\
\hline ES ... & & & & $28,5^{* *}$ & & \\
\hline F relatif à toutes les & & & & & & \\
\hline variables......... & & 32 & 35 & 52 & 43 & 9 \\
\hline$R^{2} \ldots$ & 0,787 & 0,786 & 0,798 & 0,884 & 0,640 & 0,257 \\
\hline Écart-type rés. $s^{\prime} \mathbf{R}$ & 0,910 & 0,990 & 0,770 & 0,740 & 1,380 & 1,530 \\
\hline$v_{2} \ldots \ldots \ldots \ldots \ldots$ & 35 & 35 & 35 & 34 & 101 & 101 \\
\hline
\end{tabular}

L'estimation du rendement à 8 jours est aussi bonne que celle du rendement frais dans la laiterie $\mathrm{A}$. Dans la laiterie $\mathrm{B}, 1$ 'estimation à 8 jours est nettement moins bonne. Pour expliquer les écarts observés entre les 2 laiteries nous avons pensé qu'il était intéressant de prendre en considération une variable supplémentaire : le poids moyen des fromages au démoulage et à 8 jours $\left(\mathrm{P}_{1}\right.$ et $\left.\mathrm{P}_{2}\right)$ avec les rendements correspondants $\left(R_{1}\right.$ et $\left.R_{2}\right)$. L'analyse de régression multiple (tabl. 5 ) met en évidence une relation très significative de $P_{1}$ et $P_{2}$ avec le rendement dans la laiterie $B$, alors que dans la laiterie $A$, seul le $P_{2}$ présente un " effet " significatif. On constate également que, dans la laiterie $B$, l'introduction du poids moyen des fromages a réduit sensiblement la signification de l'influence du taux de calcium. En dernière analyse, la prise en considération de $\mathrm{P}_{1}$ et de $\mathrm{P}_{2}$ a permis d'obtenir une aussi bonne estimation du rendement dans la laiterie $B$ que dans la laiterie $A$ puisqu'on arrive à expliquer ainsi entre 79 et 92 p. Ioo des variations du rendement en fromage.

Il est assez difficile de discerner dans les variations du poids moyen des fromages, la part due aux variations de composition du lait et celle due à la technique de fabrication. Toutefois, lorsque cette technique permet de réduire la variabilité du poids moyen des fromages, on estime avec plus de précision le rendement en fromage compte tenu uniquement de la composition du lait mis en œuvre.

Indépendamment du poids moyen et de l'extrait sec des fromages, il existe entre les 4 variables qui caractérisent la composition du lait, des corrélations non nulles. Bien que le modèle ne soit pas orthogonal, 1'analyse de régression multiple permet cependant de classer nettement l'importance des différentes variables que nous allons maintenant étudier séparément. 
TABLEAU 5

Analyse de la régression multiple à 5 ou 6 variables indépendantes Signification des coefficients $\left(t^{2}\right)$

\begin{tabular}{|c|c|c|c|c|c|c|}
\hline \multirow{2}{*}{ Variables } & \multicolumn{4}{|c|}{ Laiterie A } & \multicolumn{2}{|c|}{ Laiterie B } \\
\hline & $\mathrm{R}_{1}$ & $\mathrm{R}_{2}$ & $\mathrm{R}_{2}^{\prime}$ & $\mathrm{R}_{2}$ & $\mathrm{R}_{1}$ & $\mathrm{R}_{2}$ \\
\hline TB... & 1,5 & 1,1 & 2,8 & 1,9 & 0,1 & 0,4 \\
\hline TP... & $28,5^{* *}$ & $29,8^{* *}$ & $17,7 * *$ & $31,7 * *$ & $61,5^{* *}$ & $45,4^{* *}$ \\
\hline $\mathrm{TCa} \ldots \ldots \ldots$ & 0,3 & 0 & 3,6 & 1,2 & $23,3^{* *}$ & $10,5 * *$ \\
\hline $\operatorname{MAC} \ldots \ldots \ldots \ldots \ldots$ & 0,8 & 0,7 & 2,4 & 0,1 & 3,2 & $4,5^{*}$ \\
\hline $\mathrm{P}_{1}$ ou $\mathrm{P}_{2} \ldots \ldots \ldots$ & 1,4 & $33,0^{* *}$ & $7,9 * *$ & $13,2^{* *}$ & $159,0^{* *}$ & $453,0^{* *}$ \\
\hline $\begin{array}{l}\mathrm{ES} \\
\text { Test } \mathrm{F} \text { pour toutes }\end{array}$ & & & & 9,8 & & \\
\hline les variables.... & 27 & & 35 & 61 & 120 & 127 \\
\hline $\mathrm{R}^{2} \ldots \ldots \ldots \ldots \ldots$ & 0,796 & 0,892 & 0,836 & 0,917 & 0,857 & 0,864 \\
\hline$s^{\prime}{ }_{R} \ldots \ldots \ldots \ldots \ldots$ & 0,910 & 0,710 & 0,700 & 0,630 & 0,670 & 0,480 \\
\hline$v_{2} \ldots$ & 34 & 34 & 34 & 33 & 100 & 100 \\
\hline
\end{tabular}

TABLEAU 6

Ensemble des équations de régression

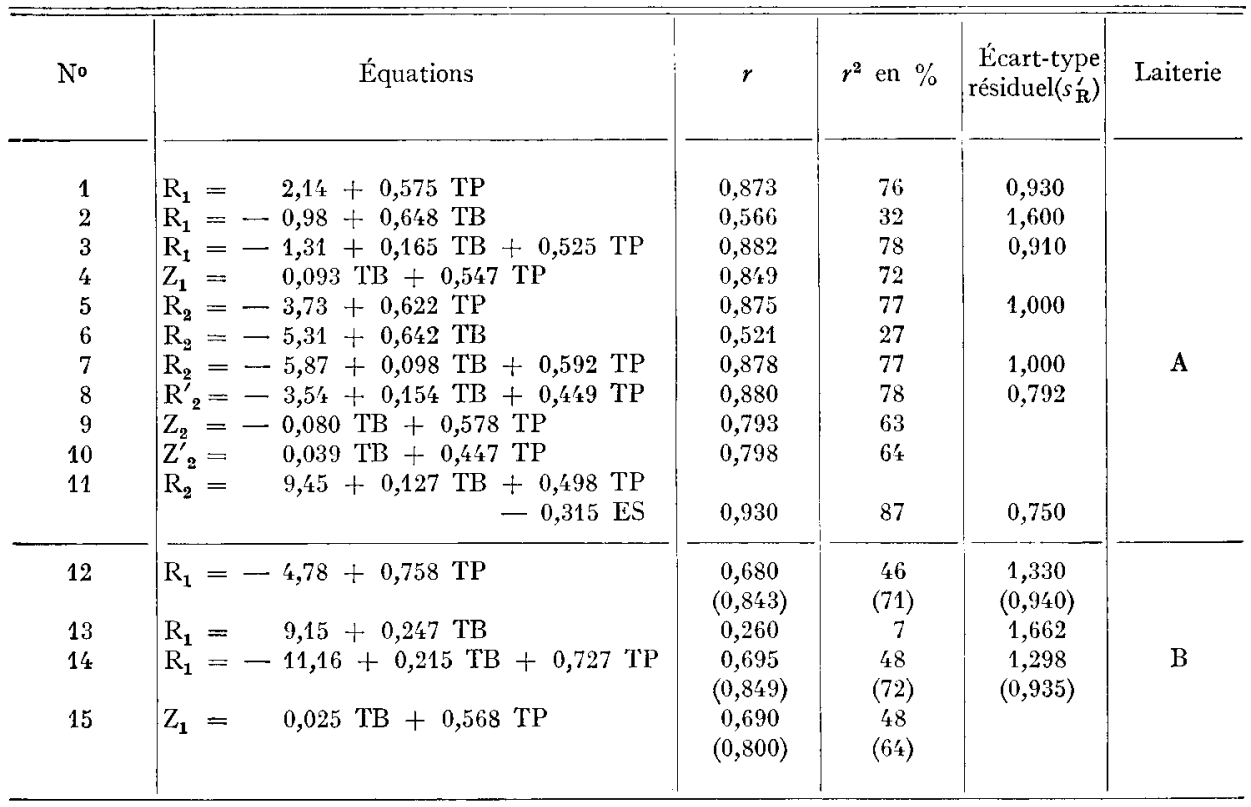

Pour la laiterie $B$, nous avons indiqué entre parenthèses les valeurs obtenues au cours des 2 dernières années de contrôles. 
I. Rendement en fromage et richesse en protéines $d u$ lait.

Des 4 variables prises en considération, le taux de protéines apparaît comme la meilleure base d'estimation du rendement en fromage. La corrélation entre le rendement frais et le TP est de 0,87 dans la laiterie A (équation $I$, tableau 6) et de 0,68 dans la laiterie $B$ (équation I2). Fn fait, dans cette dernière laiterie on observe des variations annuelles importantes comme l'indique le tableau 7 : la corrélation est de 0,84 pour les deux années I 964 et I965 et seulement de 0,63 pour les années I962 et 1963 .

TABLEAU 7

Différences anmuelles observées dans la laiterie $B$

a) moyennes arithmétiques

\begin{tabular}{|c|c|c|c|c|c|c|c|}
\hline & $\mathbf{T B}$ & $\mathrm{TP}$ & $\mathrm{TCa}$ & MAC & $P_{1}$ & $\mathrm{R}_{1}$ & $\mathrm{R}_{2}$ \\
\hline 1962 & $3^{\prime}, 9$ & 29,5 & 1,21 & 68,8 & 146 & 18,6 & 11,8 \\
\hline 1963 & 35,3 & 29,8 & 1,19 & 69,4 & 146 & 18,3 & 12,2 \\
\hline $196 \dot{t}$ & 35,1 & 30,0 & 1,18 & 70,1 & 138 & 17,5 & 11,5 \\
\hline 1965 & 34,3 & 29,7 & 1,14 & 70,0 & $13^{\prime}$ & 17,0 & 12,0 \\
\hline
\end{tabular}

b) corrélations

\begin{tabular}{|c|c|c|c|c|c|}
\hline & $\mathrm{TB}$ & $\mathrm{TP}$ & $\mathrm{TCa}$ & MAC & $\mathrm{R}_{1}$ \\
\hline $\mathrm{TP}$ & $\begin{array}{l}0,40 \\
0,16\end{array}$ & & & & \\
\hline $\mathrm{TCa}$ & $\begin{array}{l}0,58 \\
0,51\end{array}$ & $\begin{array}{l}0,51 \\
0,34\end{array}$ & & & \\
\hline $\mathrm{MAC}$ & $\begin{array}{l}0,29 \\
0,12\end{array}$ & $\begin{array}{l}0,65 \\
0,40\end{array}$ & $\begin{array}{l}0,17 \\
0,22\end{array}$ & & \\
\hline $\mathrm{R}_{1}$ & $\begin{array}{l}0,35 \\
0,26\end{array}$ & $\begin{array}{l}0,84 \\
0,68\end{array}$ & $\begin{array}{l}0,6.3 \\
0,15.5\end{array}$ & $\begin{array}{l}0,63 \\
0,20\end{array}$ & \\
\hline $\mathrm{P}_{1}$ & $\begin{array}{l}0,17 \\
0,10\end{array}$ & $\begin{array}{l}0,50 \\
0,28\end{array}$ & $\begin{array}{l}0,50 \\
0,{ }^{\prime}{ }^{\prime} 1\end{array}$ & $\begin{array}{r}0,33 \\
.0,12\end{array}$ & $\begin{array}{l}0,82 \\
0,78\end{array}$ \\
\hline
\end{tabular}

Chiffre supérieur : les 2 dernières années $190^{\prime}$ et $65(n=58)$

Chiffre inférieur : ensemble des' 1 années de 1962 à $65(n=106)$

L'estimation de l'écart-type résiduel (tabl. 6) après prise en compte de la régression sur le TP, est de $0,930 \mathrm{~kg}$ de fromage (pour roo $\mathrm{kg}$ de lait) dans la laiterie A et de $\mathrm{r}, 330 \mathrm{~kg}$ pour la laiterie $\mathrm{B}$ (dans cette dernière laiterie, il est seulement de $0,940 \mathrm{~kg}$ pour les années I964 et 65).

Dans la laiterie B, les observations ont commencé en I962, lors de la création de la coopérative, aussi les premiers résultats sont-ils peu homogènes. D'autre part, 
en 4 années, les opérations de caillage, de mise en moules et d'égouttage, ont été plus ou moins modifiées, ce qui peut expliquer les variations annuelles observées dans cette laiterie.

Dans la laiterie A, les analyses de fromage permettent de constater que l'augmentation du taux de protéines du lait entraîne (en plus d'une augmentation de rendement) une baisse de l'extrait sec des fromages, mais la corrélation observée $(r=-0,35)$ est peu significative. Quand la richesse en protéines du lait augmente, la richesse en graisse augmente relativement moins (la corrélation existant entre le TP et le rapport protéines/graisse des laits de fabrication est de 0,83 pour la laiterie A et de 0,6 I pour la laiterie B). De ce fait, on obtient plus de fromage, contenant légèrement plus d'eau et moins de graisse dans l'extrait sec.

Nous n'avons pas trouvé une corrélation étroite entre le 'TB du lait et le rapport $\mathrm{D} / \mathrm{S}$ des fromages $(r=0,20$ laiterie $\mathrm{A} ; r=0,42$ laiterie $\mathrm{B}, 25$ données recueillies en I965). Mais il convient de rappeler que ce rapport n'est qu'une valeur approchée de la teneur en graisse des fromages et que, d'autre part, les analyses concernent seulement 3 fromages pris au hasard sur chacun des lots.

\section{Rendement et taux butyreux.}

Dans tous les cas, la relation entre le taux butyreux et le rendement en fromage est moins étroite que celle qui existe entre le taux de protéines et le rendement. La corrélation positive existant entre le 'TB et le 'TP du lait est assez faible pour des laits de mélange ( $r=0,53$ et $0, \mathrm{I} 6$ pour les laiteries A et $\mathrm{B}$ respectivement, tab1. 3 ) mais tout à fait comparable à celle observée entre les teneurs moyennes pondérées des lactations de chèvres (MFyer, BUCHEGGER et GALI, I965; Ricordeau et BOUIILON, I967).

\section{Rendement, taux de calcium et matières azotées coagulables.}

Le taux de calcium est en relation étroite avec le rendement : $r=0,72$ laiterie A et $r=0,65$ laiterie B (tabl. 3). Cette liaison peut traduire l'influence directe du taux de protéines (la corrélation entre le 'TP et le $\mathrm{TCa}$ est respectivement de 0,76 et 0,34 dans les laiteries A et B). Cependant, l'analyse de régression multiple (tabl. 4) montre une influence très significative du taux de calcium dans la laiterie $B$, dans laquelle on observe (tabl. 7 a) une baisse annuelle des rendements frais associée à une baisse annuelle de la teneur moyenne des laits en calcium. L'influence du taux de calcium sur le rendement en fromage ne se retrouve pourtant pas dans la laiterie A (tab1. 4).

La proportion de matières azotées coagulables est en corrélation positive avec la richesse en matière grasse, en protéines et en calcium du lait (tabl. 3) ; elle est en corrélation positive avec le rendement, mais la liaison n'est pas très significative $(r=0,4 \mathrm{I}$ et 0,20$)$. Il faut noter également l'existence d'une corrélation négative $(r=-0,46)$ avec la teneur en extrait sec des fromages.

4. Estimation du rendement en fromage à partir du taux de protéines et du taux butyreux.

a) Équation de régression double.

Nous venons de voir que le TP est l'élément qui fournit la meilleure base pour l'estimation du rendement. Mais il reste à savoir si l'on peut se contenter de cet élé- 
ment ou s'il est préférable de tenir compte également du taux butyreux. Quant au TCa et à la MAC ce sont 2 éléments trop difficiles à doser de façon systématique pour qu'il soit bien utile de les envisager.

Les équations de régression doubles sont inscrites dans le tableau 6. Pour la laiterie $\mathrm{A}$, le test du coefficient de régression relatif au TB (tab1. 8) n'est pas signi-

\section{TABLEAU 8}

Analyse de la régression multiple à 2 ou 3 variables indépendantes

Signification des coefficients $\left(t^{2}\right)$

\begin{tabular}{|c|c|c|c|c|c|}
\hline \multirow{2}{*}{ Variables } & \multicolumn{4}{|c|}{ Laiterie A } & \multirow{2}{*}{$\frac{\text { Laiterie } B}{R_{1}}$} \\
\hline & $\mathrm{R}_{1}$ & $\mathrm{R}_{2}$ & $\mathrm{R}_{2}^{\prime}$ & $\mathrm{R}_{2}$ & \\
\hline $\mathrm{TB}$ & 2,5 & 0,7 & 2,9 & 2,2 & $9,2 * *$ \\
\hline … & $76,0^{* *}$ & $80,5^{* *}$ & $73,5 * *$ & $89,8^{* *}$ & $66,2^{* *}$ \\
\hline$\ldots$. & & & & $29,6 * *$ & \\
\hline Test $\mathbf{F}$ relatif à toutes & & & & & \\
\hline les variables $\ldots \ldots$ & & 62 & 83 & 64 & 67 \\
\hline $\mathrm{R}^{2} \ldots \ldots \ldots \ldots \ldots \ldots$ & 0,778 & 0,771 & 0,775 & 0,87 í & 0,480 \\
\hline Écart-type résiduel $s_{\mathbf{R}}^{\prime}$ & 0,910 & 1,000 & 0,792 & 0,750 & 1,298 \\
\hline$v_{2} \ldots \ldots \ldots \ldots \ldots$ & 37 & 37 & 37 & 36 & 103 \\
\hline
\end{tabular}

ficatif $\left(t^{2}=2,49\right.$; table $\mathrm{F}$ pour $v_{1}=\mathrm{I}$ et $\left.v_{2}=37\right)$; pour la laiterie $\mathrm{B}$, le test relatif au même coefficient est très significatif $\left(t^{2}=9,2\right.$; table $\mathrm{F}$ pour $\nu_{1}=\mathrm{I}$ et $\nu_{2}=$ I03).

Il est intéressant de comparer les équations obtenues dans les 2 laiteries (la variance résiduelle des rendements est supérieure dans la laiterie B mais le rapport des variances n'est pas très significatif). L'analyse de covariance permet de conclure que le parallélisme des plans de régression est acceptable. Dans cette hypothèse, on obtient une équation de forme $\mathrm{R}_{1}=\mathrm{C}^{\mathrm{te}}+0, \mathrm{I} 5 \mathrm{~TB}+0,62 \mathrm{TP}$, le coefficient relatif au TB étant significatif $\left(t^{2}=6,6\right.$ pour $\left.v=\mathrm{I} 42\right)$. Pour obtenir une estimation correcte $\mathrm{du}$ rendement, il est donc utile de faire figurer explicitement le taux butyreux dans la formule de régression commune. lait.

b) Production de fromage par $\mathrm{kg}$ de matière grasse et rapport protéines/graisse du

Au lieu d'estimer le rendement en fromage pour Ioo $\mathrm{kg}$ de lait à partir d'une équation de régression multiple, on peut calculer le rendement en fromage pour I $\mathrm{kg}$ de matière grasse utilisée $(Z)$ et étudier ensuite les variations de $Z$ en fonction du rapport protéines/matière grasse. Cette solution, préconisée par McDowal, (I936) a été développée ailleurs (Mocouot, RicordEAU et AURIoL, I962). Les équations obtenues (équations 4,9 et $\mathrm{I}_{5} \mathrm{du}$ tableau 6 ) sont tout à fait comparables à celles obtenues à partir des équations de régression double. 


\section{c) Estimation du rendement après correction pour l'extrait sec du fromage.}

Comme nous l'avons vu précédemment, à partir des résultats enregistrés dans la laiterie $A$, il existe une corrélation négative entre le rendement et l'extrait sec des fromages. Pour éliminer les variations dans la teneur en extrait sec des fromages nous avons ramené les productions de fromage à un extrait sec moyen de 42,92 p. Ioo en utilisant la formule de MAUBors et Mocovot ( 1967 ). Cette correction permet d'améliorer la précision de l'estimation du rendement : l'écart-type résiduel passe de $\mathrm{I}, 0$ (équation 7) à 0,792 (équation 8).

La prise en considération de l'extrait sec dans une équation de régression multiple $\mathrm{R}_{2}=f$ (TP, TB, ISS) permet d'obtenir une précision encore supérieure. L'équation II ainsi obtenue explique $87 \mathrm{p}$. Ioo des variations du rendement en fromage (1'écart-type résiduel est seulement de $0,75^{\circ}$ contre 0,792 avec l'équation 8 ). Ce résultat est donc très satisfaisant et mérite d'être confirmé par un plus grand nombre d'observations.

\section{DISCUSSION}

Le rendement en fromage dépend de la compétence professionnelle du fromager, des techniques de fabrication et de la composition du lait.

Le rôle du fromager est difficile à apprécier, d'autant plus que le travail du lait de chèvre est encore empirique et artisanal.

I a technique fromagère joue un rôle important et nous avons soulignél'intérêt d'uniformiser les fabrications (poids moyen des fromages et extrait sec) pour améliorer la précision des estimations du rendement d'après la richesse du lait.

Quant à la composition du lait notre étude a montré que :

I $^{0}$ La proportion de matières azotées coagulables est nettement plus faible dans le lait de chèvre (69 p. I0o) que dans le lait de vache (75 p. Ioo, Mocouot et al, Ig63) ;

$2^{\circ}$ La connaissance du seul taux de protéines permet d'expliquer jusqu'à 76 p. Ioo des variations du rendement en fromage : dans la laiterie A, on explique $87 \mathrm{p}$. Ioo des variations $\mathrm{du}$ rendement en ramenant tous les fromages obtenus en pratique à un fromage " moyen " possédant toujours la même teneur en extrait sec.

Ces données ont été obtenues en laiterie, sur des laits de producteurs récoltés tout au long de l'année. En travaillant sur des laits de petit mélange, écrémés ou triés en fonction de leur richesse en protéines, PorTmans (I967) obtient des résultats assez comparables : taux de MAC de $69,5 \mathrm{p}$. roo - équation de rendement $Z_{2}=0,093 \mathrm{~TB}+0,380 \mathrm{TP}$.

En ce qui concerne la sélection, ces résultats sont essentiels car ils permettent de préconiser la substitution de l'analyse des protéines à celle de la matière grasse. Cette substitution apparaît souhaitable du point de vue technologique, méthodologique et génétique.

Point de vue technologique. - Par rapport au lait de vache, le lait de chèvre est nettement moins "fromager " puisque le rapport protéines/graisse et la proportion de matières azotées coagulables sont plus faibles dans le lait de chèvre. Ainsi, pour 
une même richesse en graisse, le $\mathrm{kg}$ de lait de chèvre contient 2,6 à 3,0 g de protéines coagulables en moins (tab1. 9). Cela explique pourquoi les fromages de chèvres ont un " gras/sec " élevé, bien que le lait utilisé soit relativement pauvre en matière grasse.

\section{TABLEAU 9}

Correspondance entre la proportion de matières azotées coagulables du lait de Chèzre et du lait de Vache de même richesse en matière grasse

(teneur en $\mathrm{g} / \mathrm{kg}$ de lait)

\begin{tabular}{|c|c|c|}
\hline & Lait de Vache & Lait de Chèvre \\
\hline $\begin{array}{l}\text { Pour un } \mathrm{TB} \text { de } 35 \mathrm{~g} \text { on a en moyenne : } \\
\text { matières azotées totales } \ldots \ldots \ldots \ldots \\
\text { matières azotées coagulables............ }\end{array}$ & $\begin{array}{c}31,6 \\
23,7 \\
(31,6 \times 75 \%\end{array}$ & $\begin{array}{c}29,8 \text { (laiterie B) } \\
20,7 \\
(29,8 \times 69,6 \%\end{array}$ \\
\hline $\begin{array}{r}\text { Pour un TB de } 30,5 \mathrm{~g} \text { on a en moyenne : } \\
\text { matières azotées totales } \ldots \ldots \ldots \ldots \ldots \\
\text { matières azotées coagulables............. }\end{array}$ & $\begin{array}{l}30,0 \\
22,5 \\
(30,0 \times 75 \%)\end{array}$ & $\begin{array}{l}29,0 \text { (laiterie A) } \\
19,9 \times 68 \%) \\
(29,0 \times 68,7 \%)\end{array}$ \\
\hline
\end{tabular}

Pour garantir les qualités gustatives des fromages, la législation exige un "gras/sec" d'au moins $45 \mathrm{p}$. roo, ce qui nécessite environ $26 \mathrm{~g}$ de matière grasse par $\mathrm{kg}$ de lait mis en œuvre. Or, le taux butyreux moyen pondéré des chèvres contrôlées en France est de 33,9 g par kg (Disset, RicordeaU, CatTin-Vidal, r966). Même si la bonne qualité des fromages requiert un lait à plus de $26 \mathrm{~g}$ de matière grasse, les laits de mélange transformés par les laiteries sont encore excédentaires en graisse.

La richesse en matière grasse des laits n'est donc pas le facteur limitant le rendement et la qualité des fromages, aussi il n'y a pas lieu de maintenir le contrôle du taux de matière grasse des laits individuels tel qu'il est pratiqué pour les bovins laitiers. Ce contrôle conduirait finalement à une sélection "beurrière " et risquerait d'obliger - ce qui n'est pas souhaitable car l'utilisation de la crème de lait de chèvre ne semble pas à envisager - à un écrémage partiel du lait en vue de fabriquer un fromage dont la teneur en graisse ne soit pas excessive.

Point de vue méthodologique. - La suppression de l'analyse de matière grasse à la ferme doit simplifier le travail des contrôleurs laitiers. En effet, la teneur en protéines étant moins variable (d'une traite à l'autre ou entre jours de contrôles), on peut éviter le mélange proportionnel des laits du soir et du matin (opération fastidieuse et comportant des risques d'erreurs) et peut-être même réduire le nombre de prélèvements.

Point de vue génétique. - On ne possède pas encore de données précises sur l'héritabilité des constituants du lait de chèvre (RoNNINGEN en I965, trouve une héritabilité plus faible pour le taux butyreux que pour la quantité de lait : o, Io contre 0,40 ) mais les résultats obtenus par l'un d'entre nous (RICORDEAU et BouILLON, I967) permettent de penser que le taux de protéines dépend moins des conditions de milieu que le taux butyreux. De toute façon, comme il existe vraisemblablement une 
corrélation génétique positive entre les teneurs en matière grasse et en protéines, la sélection sur le seul taux de protéines doit également se traduire par une augmentation plus ou moins sensible du taux de matière grasse. On ne risque donc pas d'obtenir des laits trop riches en protéines et trop pauvres en graisse.

L'amélioration de la richesse en protéines des laits de chèvres étant un objectif souhaitable, il importe maintenant de définir une méthode de paiement susceptible de donner satisfaction, à la fois aux producteurs et aux transformateurs.

Ręu pour publication en janvier 1967.

\section{REMERCIEMENTS}

Nous tenons à remercier M. Debouver (Coopérative d'Anjouin, Indre) de l'aide qu'il nous a apportée pour ce travail, M. MaUboIs (Laboratoire de Technologie laitière, Rennes) pour ses suggestions dans l'élaboration du programme d'interprétation, Mme TASSENCOURT (Station centrale de Génétique animale) qui a participé à l'étude préliminaire et la Fédération nationale des Éleveurs de Chèvres $(36$, rue Fontaine, Paris) qui a contribué financièrement à la réalisation de l'essai dans les deux laiteries.

\section{SUMMARY}

INFLUENCE OF SEASONAL VARIATIONS IN GOAT'S MILK COMPOSITION ON CHEESE YIELD, AND ITS PRACTICAL, CONSEQUENCES ON SELECTION

A study was undertaken in two dairies (during 1965 in dairy A and from 1962 to 1965 in dairy B) :

$1^{0}$ To analyse the seasonal variations of milk composition concerning fat, total protein (total nitrogen $\times 6.38$ ), proteins which are clotted by rennet and calcium.

$2^{0}$ To determine, by the method of multiple regression, the respective influence of these constituents on cheese yield (weight of cheese obtained from $100 \mathrm{~kg}$ of milk).

Goat's milk is as rich in calcium ( $\mathrm{f}, 17 \mathrm{~g}$ per $\mathrm{kg}$ ) as cow's milk. But it is comparatively poor in total protein and proteins which are clotted by rennet : $29,38 \mathrm{~g}$ of total protein per $\mathrm{kg}$ of milk, 69, I per cent of which are clotted by rennet (table $\mathrm{r}$ ).

In both dairies, the percent total protein in milk represented the best base for estimating the cheese yield, since it explains about 75 per cent of the variations of this cheese yield; the influence of the percent fat is of little significance, if any (tables 4,6 and 8).

Some differences in findings between the two dairies are due to differences in cheesemaking technique. In dairy A for instance, the preliminary drainage of the whey, which was done when the curd was in sacks, reduced the variation of the average weight of the cheese and a better estimation of the cheese yield was possible (table 5).

To genetically improve the cheese vielding capacity of goat's milk, it is necessary to replace the butterfat determination which is now in force by a determination of the total protein content of milk.

\section{RÉFÉRENCES BIBLIOGRAPHIQUES}

Atriol P., Mocolot i., 1962. Quelques facteurs de variation de la teneur en calcium des laits de vache. I. Dairy Res., 29, $181-189$.

Disset R., Ricordent (i., Cattix-Vidal P., 1966. J'erfornances laitières des chèvres contrôlées en 1964. La Chève, 41, 8-12.

Fatoux A., 1965. La production française du lait de Chivre. Industrie lait., 1'aris, 217, 9-13. 
Fatoux A., 1966. Some figures relating to the composition of goat's milk. British Goat. Soc. Year Brok, I 8-20.

JENNESS R., 1953. Titration of calcium and magnesium in milk and milk fractions with ethylenediamine tetracetate. Analyt. Chem., 25, 966-968.

Gaillard G., Huni C., 1965. A propos du fromage de chevre. Indusirie lail., Paris, 217, i4-I 7 .

Knowles F., Watkin J. E., I938. The milk of the goat under English conditions. J. Dairy Res., 9, $153^{-16} 5$.

Labussikire J., Alais C., Bejambes M., I959. Le fromage de chèvre du Sancerrois. Industrie lait, Paris, 153 et 154, io p.

Maubois J. I.., Mocevot G., 1967. Comment ramener à la même teneur en substance sèche les fabrications de fromage en vue de comparer les rendements respectifs du lait en fromage. Industrie lait, Paris, 239, 1 5-18.

MCDOWALL F. H., 1936. The cheese yielding capacity of milk and its relation to the method of payment for milk for cheesemaking. Dairy Res. Insi. N. Z., 72, $137-364$.

Meyer J., Blchegger O., GaLL C., 1965: Ein Beitrag zur züchterischen Beeinflussung der Hilchbestandteile. Arb. Inst. Tierz. Vererb. Konstit-Forsch., Univ. Nunich (6), 46-53.

Mocevot G., Al.AIS C., 1950. Calcul du poids de lactosérum correspondant à un poids donné de lait (non publié).

Mocovot G., Ricordeau G., Auriol P., 1963. Lstimation du rendement en fromage Gruyère de Comté en fonction de la richesse du lait de chaudière. Ann. Zootech., 12, 53-66.

Mocquot G., Blanc-Patin E., Sainclivier M., Rousseaux P., Jecnet R., I953. Relation entre la teneur du lait en matière azotée et le poids de fromage obtenu. Induslrie lait, P'aris, 203, $269^{-2} 7^{6}$.

Portmann A., Vedrenne P., Vassal L., Dicruet P., Carcolet L., 1966. Fssai de conservation du lait de chèvre destiné à la fromagerie à l'état de caillé congelé. Industrie lail, Paris, 228, $13^{-1} 8$.

Portmann A., Pierre Alice, Vedrenne P., ig67. Importance de la matière grasse et de la matière azotée du lait de chèvre sur le rendement en fromage (non publié).

Ricordeau G., Bouillon J., r967. Estimation des performances laitières des chèvres à partir des lactations partielles : quantité de lait, richesse en matiére grasse et protéines. Bull. lech. Inf. Ingrs. Serv's. agric., 218, 303-310.

Rønningen K., 1965. Causes of variation in the flavour intensity of goat milk. Icla Agric. Scand., 15, $301-34^{2}$. 